\title{
Flow of Water-Oil Emulsion through an Orifice
}

\author{
Shaharin A. Sulaiman*, Mohamad Nazmi Z. Moni, and Siti Norazilah Ahmad Tamili \\ Department of Mechanical Engineering, Universiti Teknologi PETRONAS, 32610 Seri Iskandar, \\ Perak, Malaysia
}

\begin{abstract}
The oil-in-water $(\mathrm{O} / \mathrm{W})$ and water-in-oil $(\mathrm{W} / \mathrm{O})$ emulsions are two common types of emulsions found in oil production industry. While stable $\mathrm{O} / \mathrm{W}$ may be beneficial in transporting crude oil, stable $\mathrm{W} / \mathrm{O}$ poses a flow assurance problem that leads to disruptions and losses in oil production line. This study examines the behaviour of both types of emulsion (40:60, 50:50 and 60:40 water-oil emulsion, vol. basis) subjected to 3/4D, 1/2D and $1 / 4 \mathrm{D}$ orifices within a pipeline. The study confirms that oil and water may form emulsion with only mechanical agitation and dynamic flow in the pipeline and without the presence of any emulsifying agent. The flow rate and the velocity of all emulsions were found to drop with the reduction of orifice diameter.
\end{abstract}

\section{Introduction}

When two immiscible liquids, wherein droplets of one phase are encapsulated within another phase, the mixture is known as emulsion [1]. In oil production industry, there are two basic forms of emulsion: (a) oil-in-water $(\mathrm{O} / \mathrm{W})$ emulsion (dispersion and encapsulation of oil droplets within a water column), and (b) water-in-oil (W/O) emulsion (i.e. the opposite of $\mathrm{O} / \mathrm{W}$ ) [2]. In addition, there is also another form of emulsion, which is known as multiple emulsion [3]. If the water and oil become separate after a while, then the emulsion is regarded as unstable. Stable oil-in-water emulsions are formed by two main types of agents of molecular emulsifying, which are surfactants of small-molecule and polymers that are watersoluble. In complex emulsions, there often exists a multicomponent mixture of small amphiphiles (chemical compounds possessing both hydrophilic and lipophilic properties) and surface-active polymers [4]. The structure, rheology and stability of emulsion are related to the composition, viscoelasticity and thickness of the adsorbed stabilizing layer at the oilwater interface; ditto on the nature and strength of the interactions between adsorbed layers on different droplets. The more common emulsion in the oil field is $\mathrm{W} / \mathrm{O}$. Stable W/O poses a flow assurance problem leading to disruption and losses in the oil production line [5]. On the other hand, $\mathrm{O} / \mathrm{W}$ can be beneficial; for example, in transporting highly viscous crude oil [6]. The formation and stability of emulsion in oil production line is the result of high kinetic energy, which results in high shear [7], and this usually occurs at flow constrictions such as nozzle, venturi, orifice and choke. The flow of this emulsion is not well understood.

The objective of this study was to examine the flow behavior of water-oil emulsion when passing through orifice constriction of different diameters in terms of volumetric flow rate,

* Corresponding author: shaharin@utp.edu.my 
velocity and pressure drops in between the upstream and downstream of the constriction. This study is useful in understanding the effect of several controlling parameters to the flow of oil upon passing through constrictions, which are found in oil production lines.

\section{Methodology}

\subsection{Materials preparation}

The materials involved in this study were a 55-gallon (200 L) barrel of Mobil Delvac 1340 and purified water. The typical properties of Mobil Delvac 1340 are shown in Table 1 . The mixing of oil and water was done in the mixing tank according to the ratios shown in Table 2. The mixing tank was first filled with water to the required quantity by volume in order to produce the desired water-oil ratio. A propeller-type mechanical stirrer was turned on as its blade was submerged underwater during the filling process. Oil was then poured into the tank and the rise in liquid volume was continuously monitored during the process to ensure the correct mixing ratio was obtained. The volume of the liquid in the mixing tank was measured using a $2 \mathrm{~m}$ long dipstick and an ordinary measuring tape with a potential error of $\pm 2.3 \mathrm{~mm}$, or translated to $\pm 1.81 \mathrm{~L}$.

Table 1. Properties of Mobil Delvac 1340.

\begin{tabular}{|l|c|}
\hline SAE Grade & $\mathbf{4 0}$ \\
\hline Viscosity (centistokes): & \\
\hline at $40^{\circ} \mathrm{C}$ & 132 \\
\hline at $100^{\circ} \mathrm{C}$ & 14.7 \\
\hline Pour point, ${ }^{\circ} \mathrm{C}$ & -21 \\
\hline Flash point, ${ }^{\circ} \mathrm{C}$ & 256 \\
\hline Density at $15^{\circ} \mathrm{C}, \mathrm{kg} / \mathrm{L}$ & 0.892 \\
\hline
\end{tabular}

Table 2. Experimental water-oil ratio.

\begin{tabular}{|c|c|c|}
\hline Water-oil ratio & Volume of water, $\mathbf{L}$ & Volume of oil, $\mathbf{L}$ \\
\hline $40: 60$ & 133 & 200 \\
\hline $50: 50$ & 200 & 200 \\
\hline $60: 40$ & 300 & 200 \\
\hline
\end{tabular}

\subsection{Experimental rig}

Figure 1 shows the experimental flow loop rig used in this study. The experimental rig consisted of two cylindrical tanks with $2000 \mathrm{~L}$ maximum capacity and a series of 1 " OD pipe spools, all constructed from grade 316L stainless steel. Also shown in Figure 1 are the rotameter, pump and other measuring instruments. The two tanks each served as a mixing tank, in which the emulsion was prepared with the help of a propeller-type mechanical stirrer. The tank also functioned as a settling tank, for which the emulsion was left to settle after the end of each experiment. The longest straight pipe section is $10 \mathrm{~m}$, where $5 \mathrm{~m}$ of the pipe was located upstream of orifice station and $5 \mathrm{~m}$ at the downstream. The $5 \mathrm{~m}$ length at the upstream was of length-to-diameter ratio of 197, which was sufficient for the flow to fully develop.

Two pressure taps were located near the end of the upstream and downstream pipe section, where 10-bar spring pressure gauges were placed. An ultrasonic velocity profiler was positioned near the downstream pressure gauge to evaluate the velocity and the volume flow 
rate of the liquid at the downstream. The orifice station was equipped with three orifice plates of different diameters, as shown in Table 3, which could be placed interchangeably for the microemulsions study. The orifice station was also equipped with four tap points for manometer readings that enabled the reading of the irrecoverable pressure loss caused by the orifice constriction. The location details of the tap points are shown in Figure 2.

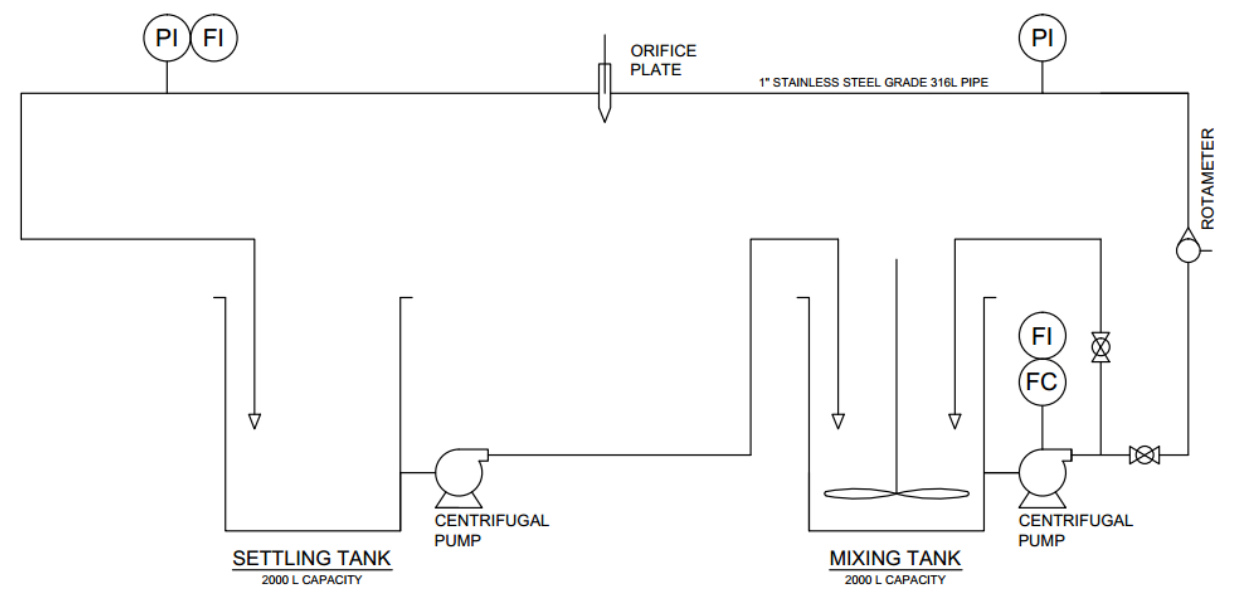

Fig. 1. Experimental flow loop rig. Note: FC: flow controller; FI: flow indicator; PI: pressure indicator.

Table 3. Type of orifice plates and their diameter.

\begin{tabular}{|c|c|c|}
\hline Orifice type & Description & Diameter, in \\
\hline 1/4 ID & $1 / 4$ of pipe ID & 0.7868 \\
\hline 1/2 ID & $1 / 2$ of pipe ID & 0.5245 \\
\hline 3/4 ID & $3 / 4$ of pipe ID & 0.2623 \\
\hline
\end{tabular}

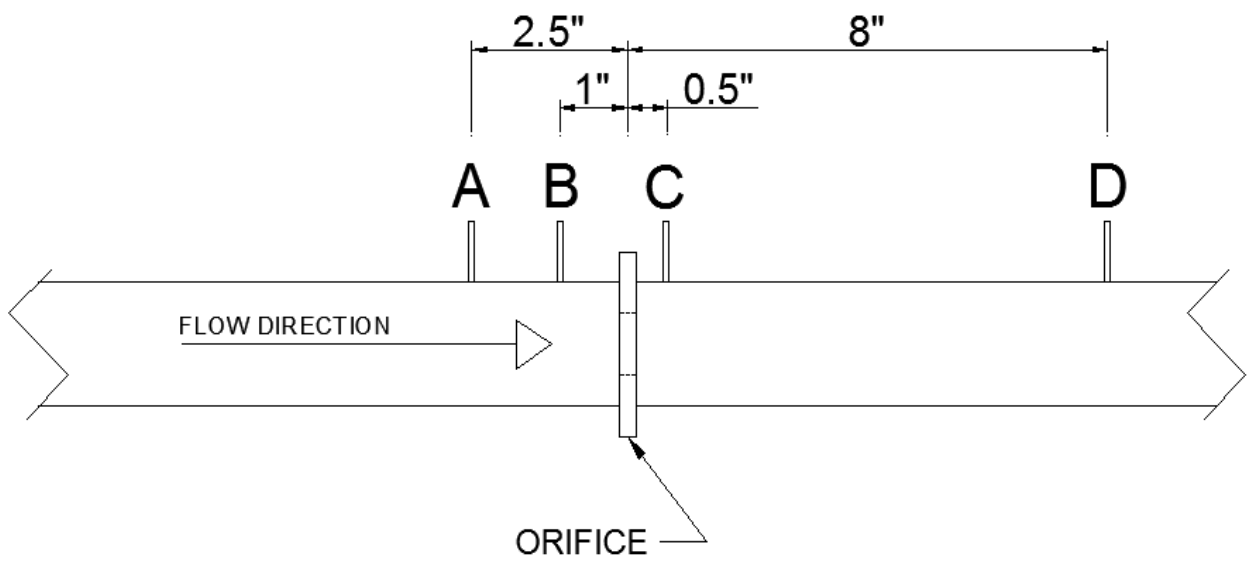

Fig. 2. Location details of tap points A, B, C and D 
A centrifugal pump, which was equipped with a speed controller and a pressure gauge, was located at the downstream to avoid the effect of pump on flow pattern of emulsion. A rotameter was placed slightly after the pump to measure the flow rate, which was regulated by manipulating a ball valve positioned before the rotameter. Excess liquid was purged back into the mixing tank through a bypass pipe to avoid excessive pressure on the pump. The emulsion was channelled into the settling tank to collect and settle after each experiment. It could also be transferred back into the mixing pump by using another centrifugal pump dedicated for emulsion transfer process.

\subsection{Experiment procedure}

The premixed emulsion in the mixing tank was maintained in its emulsion state by continuous stirring and recirculating using the centrifugal pump through the bypass pipe for around 1520 minutes until homogeneous liquid was observed in the tank and at the outlet of the bypass pipe. In the meantime, the desired orifice plate to be used was placed at the orifice station, and the ultrasonic flow meter was turned on. The flow was rechannelled into the main pipeline by adjusting the ball valve. Since the centrifugal pump used was able to self-regulate the flow according to its intake pressure, the intake pressure was set to 1 bar. Pressure, velocity and flow rate readings were taken once the emulsion flowed through the main pipeline and into the settling tank. The centrifugal pump would automatically shut down once low intake pressure was detected, indicating the low level of remaining emulsion in the mixing tank. The emulsion was left to settle in the settling tank for about 30 minutes. Three consecutive runs were conducted for each experiment for consistency.

\section{Results and discussions}

The emulsion was observed as stable as the oil and water became separated after about 10 minutes. Shown in Fig. 3 is the variation of differential pressure across points A and D for different water-oil emulsions and orifice sizes. It is shown in Fig. 3 that the smaller orifice size, the higher is the pressure drop; this is normal for flow through orifice. Obviously, the mixture with lowest amount of water (40\%) has the least pressure drop. The other two mixtures are shown to be having similar pressure drops, which are outstandingly higher than that of $40 \%$ water. A similar trend is shown in Fig. 4, although the pressure drop is shown to be slightly higher; this has been anticipated due to the fact that points $\mathrm{B}$ and $\mathrm{C}$ are the zone where the flow is in transition stage due to their proximity with the orifice.

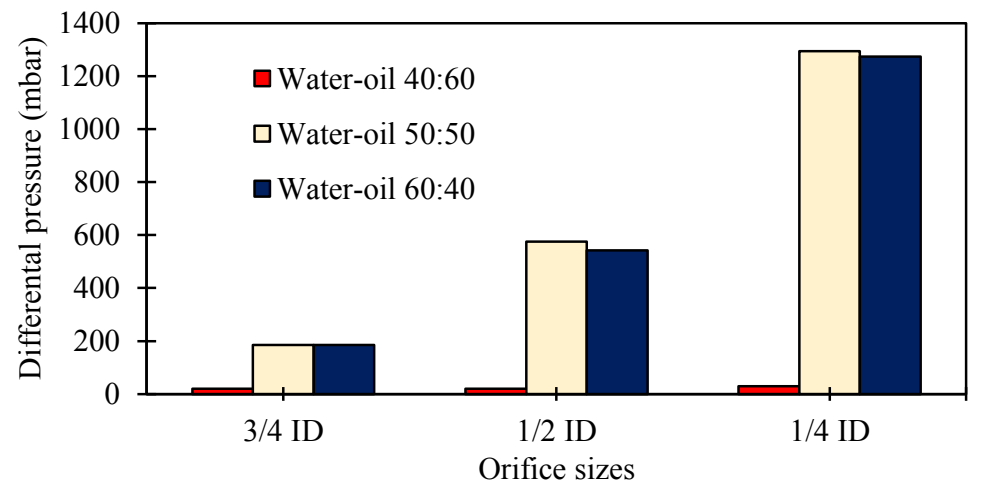

Fig. 3. Pressure differences across points A-D for different water-oil emulsions and orifice sizes. 


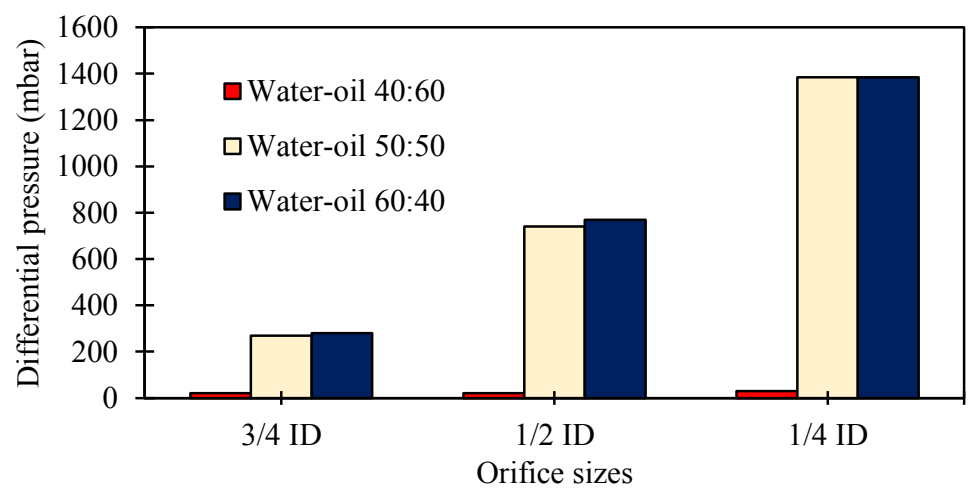

Fig. 4. Pressure differences across points B-C for different water-oil emulsions and orifice sizes.

The variation of volume flow rate at the downstream point of the pipe for different wateroil emulsions and orifice sizes is shown in Fig. 5. It is shown in Fig. 5 that the smaller the orifice size, the smaller would be the resulting flow rate. This is expected so because the smaller orifice opening would restrict flow and high amount of head losses would be expected. As for the emulsions, high water content contributed to higher flow rates at all orifice sizes. This is fairly due to the diluted properties of the emulsion with high water content that significantly reduced its viscosity, thus causing the emulsion to flow much freely compared to those with higher oil content.

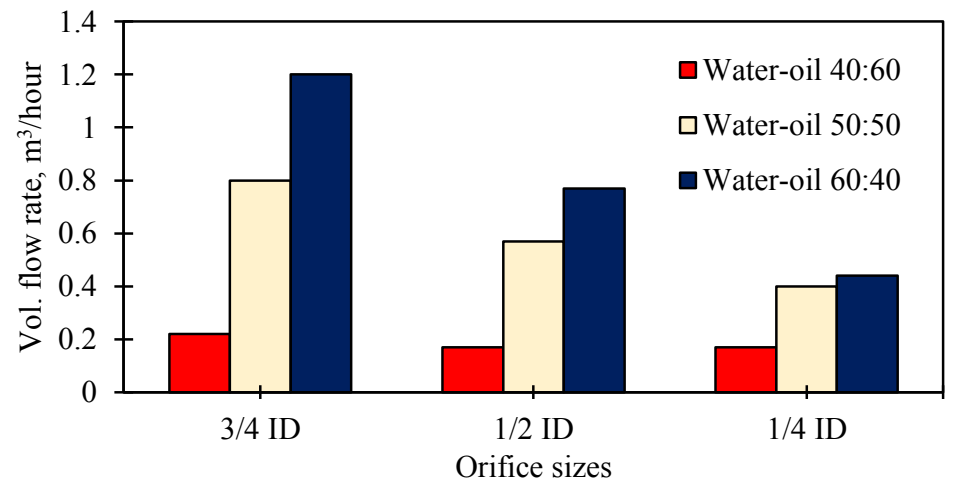

Fig. 5. Downstream volumetric flow rate for different water-oil emulsions and orifice sizes.

Shown in Fig. 6 is the variation of velocity at the downstream point of the pipe for different water-oil emulsions and orifice sizes. It is shown in the figure that the larger the orifice size, the higher would be the resulting flow velocity. This is in agreement with the smaller pressure drop experienced by the largest orifice. As for the emulsions, significant drops of velocity were experienced by the 50:50 and 60:40 water-oil emulsions with the reduction of the orifice size. Despite an increase in viscosity due to the increased proportion of water to oil, the emulsions were sufficiently thick to experience a significant flow resistance through the orifice with reducing diameter, thus causing the drop in velocity. 


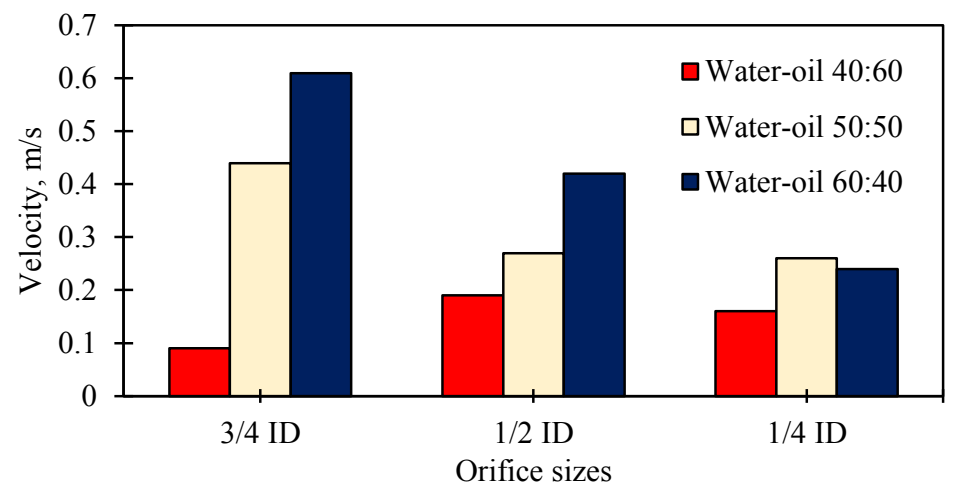

Fig. 6. Downstream velocity for different water-oil emulsions and orifice sizes.

\section{Conclusions}

From this work, the following conclusions can be made:

1. The mixture of oil and water can successfully form a stable emulsion by only mechanical agitation and without the use of any emulsifying agent;

2. The observations made from the experiments confirmed the behaviour of emulsions of varying viscosity through orifices of different diameter, where smaller constrictions were found to cause significant reduction in flow rate and velocity

3. The experimental results have contributed to the understanding of emulsion behaviour through constrictions within pipelines beneficial for improving downstream processing line particularly in the oil production industry.

Further investigation covering a wider range of emulsion, particularly those involving stable emulsion, would be required in order to further understand the emulsion flow.

This project is funded by the Ministry of Higher Education Malaysia through the Fundamental Research Grant Scheme, grant number FRGS/1/2014/TK01/UTP/02/5.

\section{References}

1. R.P. James, R.P. Charles, Petroleum Spills, 3 (1985)

2. G. Chen, D. Tao, Fuel Processing Technology, 86 (2005)

3. S. Kokal, Crude-Oil Emulsions: A State-Of-The-Art Review (SPE, Saudi Aramco Production and Facilities, 2005).

4. E. Dickinson, Pure \& App. Chem., 64, 11 (1992)

5. A.A. Umar, I.B.M. Saaid, A.A. Sulaimon, AIP Conf. Proc. 1774 (AIP Publishing, 2016)

6. S.N. Ashrafizadeh, E. Motaee, V. Hoshyargar, J. Petroleum Science and Engineering 86-87 (2012)

7. M.K. Sharma, D.O. Shah, Introduction to Macro- and Microemulsions in Macro- and Microemulsions American Chemical Society, (1985) 